\title{
Radiation by an electron in an electromagnetic plane wave: The quasiclassical formula and other domains of applications
}

\author{
X. Artru* \\ Université de Lyon, CNRS/IN2P3, UCBL, Institut de Physique Nucléaire de Lyon, \\ 69622 Villeurbanne, France
}

(Received 30 December 2018; published 31 May 2019)

\begin{abstract}
The semiclassical Baïer-Katkov (BK) formula is an approximation to the spectrum of the radiation by an ultrarelativistic electron in a nonuniform field, taking into account the recoil and spin effects. The question why it involves only the initial electron trajectory is addressed. When the field is an electromagnetic plane wave, the exact helicity amplitudes in the null-plane frame are derived using Volkov solutions of the Dirac equation and a modified form of the BK formula is obtained. In passing, the application to Compton scattering is outlined and interesting spin properties are noted. For a general field, two conditions of validity of the BK formula are proposed: (1) the field is approximately uniform in the transverse directions, (2) the relative velocity between the field and the electron is close to $c$. The applications to bremsstrahlung, coherent bremsstrahlung and channeling radiation are discussed.
\end{abstract}

DOI: 10.1103/PhysRevAccelBeams.22.050705

\section{INTRODUCTION}

The semiclassical Baïer-Katkov (BK) formula [1,2], written below in the form of Eqs. (24), (27), (28) and 29, predicts an energy-angle spectrum of the radiation by an ultrarelativistic electron in an initial nonuniform field $\mathcal{F}_{\text {in }}=\{\mathbf{E}(t, \mathbf{r}), \mathbf{B}(t, \mathbf{r})\}$. Like the classical radiation formula [3], it involves only the initial classical trajectory of the electron, $\mathbf{r}_{i}(t)$ (prolongated up to $t=+\infty$ ) but, in addition, it takes into account the loss of energy-momentum (or recoil effect) and predicts a dependence on the electron polarization. These two effects become important when the photon energy $\omega$ is comparable to the electron one, $\epsilon \equiv p^{0}=$ $\gamma m=\left(m^{2}+\mathbf{p}^{2}\right)^{1 / 2}{ }^{1}$ Here are two examples: (i) linear or nonlinear Compton effect when $p_{i} \cdot k_{i} \gtrsim m^{2}\left(p_{i}\right.$ and $k_{i}$ are the initial 4-momenta of the electron and the photon); in this case $\mathcal{F}_{\text {in }}$ is the classical field describing the initial photon or coherent state of photons; (ii) synchrotron radiation in the strong field regime, $\chi \equiv \gamma F / m^{2} \gtrsim 1$, where $\mathbf{F}=-e(\mathbf{E}+$ $\mathbf{v} \times \mathbf{B})$ is the Lorentz force. In the uniform field approximation, the natural photon energy cutoff $\omega_{c}=\chi \epsilon /(1+\chi)$ is comparable to $\epsilon$. The BK formula generalizes the "magnetic bremsstrahlung" formula [see, e.g., Eq. (90.22) of [4]] to the

\footnotetext{
x.artru@ipnl.in2p3.fr

" $\gamma$ " can also designate a photon. We use units where $\hbar=c=1 ; \alpha=e^{2} /(4 \pi) \simeq 1 / 137$.

Published by the American Physical Society under the terms of the Creative Commons Attribution 4.0 International license. Further distribution of this work must maintain attribution to the author(s) and the published article's title, journal citation, and DOI.
}

case of nonuniform fields. The BK formula has been applied, with good enough agreement with experiment [5-8], in Monte Carlo simulations of channeling radiation or coherent radiation in bent crystals at high energy (e.g., $50 \mathrm{GeV}$ for axial channeling in germanium, $10 \mathrm{GeV}$ in tungsten). Here the strong field parameter is $\chi \equiv \max \left\{e \gamma\left|\mathbf{E}\left(\mathbf{r}_{T}\right)\right| / m^{2}\right\} \gtrsim 1$, where $\mathbf{E}\left(\mathbf{r}_{T}\right)$ is the Lindhard continuous field.

The fact that the BK formula does not involve a final electron trajectory $\mathbf{r}_{f}(t)$ is surprising, since the true quantum amplitude involves both initial and final wave functions, as in Eq. (14) below. On the other hand, $\mathbf{r}_{f}(t)$ is not uniquely defined. If the emission is considered as a local process which occurs at a definite time $t_{\mathrm{em}}, \mathbf{r}_{f}(t)$ should start at the point $\mathbf{r}_{i}\left(t_{\mathrm{em}}\right)^{2}$ and, due to the recoil effect, separate from $\mathbf{r}_{i}(t)$ for $t>t_{\mathrm{em}}$. But in reality the photon source is the whole initial trajectory [see Eq. (23) or (29)]. For a given k one can only define a family of final trajectories $\mathbf{r}_{f}\left(t ; t_{\mathrm{em}}\right)$. The BK formula does not involve this family explicitly, as if $\mathbf{r}_{f}\left(t ; t_{\mathrm{em}}\right)$ was fully determined by $\mathbf{r}_{i}(t)$ for $t>t_{\mathrm{em}}$, whatever the field $\mathcal{F}_{\text {in }}(t, \mathbf{r})$ is around $\mathbf{r}_{i}(t)$.

In this article we show that the BK formula becomes exact, after a replacement of $\epsilon$ by $p^{+} / 2 \equiv\left(\epsilon+p^{z}\right) / 2$, when the field $\mathcal{F}_{\text {in }}(t, \mathbf{r})$ is a plane wave. For this purpose we will use the Volkov solutions of the Dirac equation, to which we associate a classical electron trajectory. In passing we analyze the helicity dependence of the radiation amplitude, give the Stokes parameters of the photon and apply the exact formula to Compton scattering. Then we

\footnotetext{
${ }^{2}$ Here we neglect a side slipping $[9,10]$ of the electron, necessary to conserve energy and momentum.
} 
will give examples where the BK formula is accurate. We finally discuss its use in channeling radiation.

\section{VOLKOV STATES}

Let $\mathcal{F}_{\text {in }}(t, \mathbf{r})=\{\mathbf{E}(\zeta), \mathbf{B}(\zeta)\}$ be a plane electromagnetic wave packet moving in the $-\hat{\mathbf{z}}$ direction. $\zeta \equiv X^{+}=t+z$ and $\tau \equiv X^{-}=t-z$ are the lightlike coordinates of a spacetime point $X$, while $X_{T}=(x, y)$ are the transverse coordinates. $\mathcal{F}_{\text {in }}$ can be derived from a vector potential $\mathbf{A}_{T}(\zeta)=\left(A^{x}(\zeta), A^{y}(\zeta)\right)$ :

$$
\mathbf{E}(\zeta)=d \mathbf{A}_{T}(\zeta) / d \zeta, \quad \mathbf{B}(\zeta)=-\hat{\mathbf{z}} \times \mathbf{E}(\zeta)
$$

The Dirac equation for the electron,

$$
\left[i \partial_{t}+\boldsymbol{\alpha} \cdot\left(i \nabla-e \mathbf{A}_{T}\right)-m \beta\right] \Psi(X)=0,
$$

admits the Volkov solutions (see [11] or Chapter IV in [4]), which are eigenstates of $p^{+}=p^{0}+p^{z}=i \partial_{t}-i \partial_{z}$ and $\mathbf{P}_{T}=-i \nabla_{T}$. They can be factorized in the form

$$
\Psi(X)=\exp \left\{-i p^{+} \tau / 2+i \mathbf{P}_{T} \cdot \mathbf{X}_{T}\right\} \psi(\zeta) .
$$

Then Eq. (2) reduces to

$$
\begin{aligned}
& {\left[\left(1-\alpha_{z}\right) p^{+} / 2+i\left(1+\alpha_{z}\right) \partial_{\zeta}\right.} \\
& \left.\quad-\boldsymbol{\alpha}_{T} \cdot \mathbf{p}_{T}(\zeta)-m \beta\right] \psi(\zeta)=0,
\end{aligned}
$$

where $\mathbf{p}_{T}(\zeta)=\mathbf{P}_{T}+e \mathbf{A}_{T}(\zeta)$ is the (gauge-invariant but nonconserved) mechanical transverse momentum, $\mathbf{P}_{T}$ being the (conserved) canonical one. We use a representation of Dirac matrices where

$$
\begin{aligned}
& \alpha_{z}=\left(\begin{array}{cc}
\mathbf{1} & \mathbf{0} \\
\mathbf{0} & -\mathbf{1}
\end{array}\right), \quad \beta=\left(\begin{array}{ll}
\mathbf{0} & \mathbf{1} \\
\mathbf{1} & \mathbf{0}
\end{array}\right), \\
& \boldsymbol{\alpha}_{T}=\left(\begin{array}{cc}
\mathbf{0} & \boldsymbol{\sigma}_{z} \boldsymbol{\sigma}_{T} \\
\boldsymbol{\sigma}_{T} \boldsymbol{\sigma}_{z} & \mathbf{0}
\end{array}\right),
\end{aligned}
$$

obtained from the spinorial representation [4] by permuting the second and fourth components of the spinors. Thus $\psi=\left(\begin{array}{l}\phi \\ \chi\end{array}\right)$, where the Pauli spinors $\phi$ and $\chi$ are the $\alpha_{z}=+1$ and $\alpha_{z}=-1$ components respectively. Equation (4) splits in

$$
\begin{aligned}
& 2 i p^{+} \partial_{\zeta} \phi(\zeta)=\left[m^{2}+\mathbf{p}_{T}^{2}(\zeta)\right] \phi(\zeta), \\
& p^{+} \chi(\zeta)=\left[m-\sigma_{z} \boldsymbol{\sigma}_{T} \cdot \mathbf{p}_{T}(\zeta)\right] \phi(\zeta)
\end{aligned}
$$

The solution of the first equation is

$$
\phi(\zeta)=\phi(0) \exp \left\{-i \int_{0}^{\zeta} d \zeta^{\prime} \frac{m^{2}+\mathbf{p}_{T}^{2}\left(\zeta^{\prime}\right)}{2 p^{+}}\right\} .
$$

Equations (3), (7) and (8) form the Volkov solution. We will consider the Volkov states $\left|p^{+}, \mathbf{P}_{T}, \lambda\right\rangle$, where $\phi(0)=|\lambda\rangle$ is the basic eigenvector of $\sigma_{z}$ with eigenvalue $2 \lambda . \lambda= \pm 1 / 2$. is the helicity in the null plane or $p^{+} \rightarrow \infty$ frame. These states form a continuous complete basis with the orthogonality relation,

$$
\begin{aligned}
& \left\langle p^{+}, \mathbf{P}_{T}, \lambda \mid p^{\prime+}, \mathbf{P}_{T}^{\prime}, \lambda^{\prime}\right\rangle \\
& \quad=\delta_{\lambda \lambda^{\prime}} J^{+} \times(2 \pi)^{3} \delta\left(p^{+}-p^{\prime+}\right) \delta\left(\mathbf{P}_{T}-\mathbf{P}_{T}^{\prime}\right),
\end{aligned}
$$

where $J^{+} / 2=\left(J^{0}+J^{z}\right) / 2=d N_{e} /\left(d^{2} \mathbf{X}_{T} d \tau\right)$ is the flux density crossing a hyperplane of constant $X^{+}$. With our normalization,

$$
J^{+} \equiv \Psi^{\dagger}(X)\left(1+\alpha_{z}\right) \Psi(X)=2 \phi^{\dagger}(0) \phi(0)=2 .
$$

\section{A. Associated classical trajectories}

Let us consider a classical electron world line $X_{\mathrm{cl}}(\zeta)=\left[t_{\mathrm{cl}}(\zeta), \mathbf{r}_{\mathrm{cl}}(\zeta)\right]$, parametrized by $\zeta$ instead of $t$. In the field $\mathcal{F}_{\text {in }}, p^{+}$is conserved due to the second equation of (1). The canonical transverse momentum $\mathbf{P}_{T}=\mathbf{p}_{\mathrm{cl}, T}-$ $e \mathbf{A}_{T}$ is also conserved due to the invariance of the four-potential in the transverse directions. Thus $\mathbf{p}_{\mathrm{cl}, T}(\zeta)=\mathbf{P}_{T}+e \mathbf{A}_{T}(\zeta)$. From the mechanical constraint $p^{2}=m^{2}$ we have also $p^{-}=p^{-}(\zeta)=\left[m^{2}+\mathbf{p}_{T}^{2}(\zeta)\right] / p^{+}$. Using $d X_{\mathrm{cl}}^{\mu} / d \zeta=p^{\mu} / p^{+}$, we deduce

$$
\begin{gathered}
\mathbf{r}_{\mathrm{cl}, T}(\zeta)=\mathbf{r}_{\mathrm{cl}, T}(0)+\int_{0}^{\zeta} d \zeta^{\prime} \frac{\mathbf{p}_{\mathrm{cl}, T}\left(\zeta^{\prime}\right)}{p^{+}} \\
\tau_{\mathrm{cl}}(\zeta)=\tau_{\mathrm{cl}}(0)+\int_{0}^{\zeta} d \zeta^{\prime} \frac{m^{2}+\mathbf{p}_{\mathrm{cl}, T}^{2}\left(\zeta^{\prime}\right)}{\left(p^{+}\right)^{2}} .
\end{gathered}
$$

To this trajectory corresponds the Volkov states $\mid p^{+}, \mathbf{P}_{T}$, $\pm 1 / 2\rangle$. Conversely, for one Volkov state we can associate an infinite family (or "bundle") of classical trajectories, all parallel but distinguished by their $\mathbf{r}_{\mathrm{cl}}(0)$. Since $\mathbf{p}_{\mathrm{cl}, T}(\zeta)$ is equal to the eigenvalue of the operator $\mathbf{p}_{T}(\zeta)=\mathbf{P}_{T}-$ $\mathbf{A}_{T}(\zeta)$ of the Volkov state, we will omit the subscript "cl" in the former.

\section{RADIATIVE TRANSITION BETWEEN VOLKOV STATES}

The spectrum of the radiation by an electron in a monochromatic plane wave was calculated by Ritus [12] using the Volkov states. Here we consider the more general case where the wave is not monochromatic. The radiative transition between states $|i\rangle=\left|p_{i}^{+}, \mathbf{P}_{i T}, \lambda_{i}\right\rangle$ and $|f\rangle=$ $\left|p_{f}^{+}, \mathbf{P}_{f T}, \lambda_{f}\right\rangle$ yields the differential photon spectrum per electron 


$$
\begin{aligned}
\frac{d N(\mathbf{k})}{d^{3} \mathbf{k} / \omega} & \equiv \frac{d N(\mathbf{k})}{d^{2} \mathbf{k}_{T} d k^{+} / k^{+}} \\
& =\frac{\alpha}{4 \pi^{2}} \sum_{\hat{\boldsymbol{\varepsilon}}, \lambda_{f}}\left|\left\langle\lambda_{f}|a(\mathbf{k}, \hat{\boldsymbol{\varepsilon}})| \lambda_{i}\right\rangle\right|^{2}
\end{aligned}
$$

with $\omega=|\mathbf{k}|$ and

$$
\begin{aligned}
\left\langle\lambda_{f}|a(\mathbf{k}, \hat{\boldsymbol{\varepsilon}})| \lambda_{i}\right\rangle= & \int_{-\infty}^{+\infty}(d \zeta / 2) \exp \left(i k^{-} \zeta / 2\right) \\
& \times \psi_{f}^{\dagger}(\zeta)\left(\hat{\boldsymbol{\varepsilon}}^{*} \cdot \boldsymbol{\alpha}\right) \psi_{i}(\zeta) .
\end{aligned}
$$

$\psi(\zeta)$ was defined in (3) and normalized with (10); $\hat{\boldsymbol{\varepsilon}}$ is the polarization vector of the photon $\left(\hat{\boldsymbol{\varepsilon}} \perp \mathbf{k}, \hat{\boldsymbol{\varepsilon}}^{*} \cdot \hat{\boldsymbol{\varepsilon}}=1\right)$.

The derivation of Eqs. (13) and (14) is given in the Appendix A. For a given photon momentum $\mathbf{k}$ the electron final state is fully fixed, apart from $\lambda_{f}$, by the conservation laws

$$
p_{f}^{+}=p_{i}^{+}-k^{+}, \quad \mathbf{P}_{f T}=\mathbf{P}_{i T}-\mathbf{k}_{T}
$$

which result from the invariance of $\mathcal{F}_{\text {in }}$ under translations in $\tau$ and $X_{T}$.

Let us place ourselves in a frame where $\mathbf{k}_{T}=\mathbf{0},{ }^{3}$ using the Lorentz transformation "on the null plane"

$$
\begin{aligned}
\mathbf{X}_{T} & \Rightarrow \mathbf{X}_{T}+\boldsymbol{\theta} X^{+}, \\
X^{-} & \Rightarrow X^{-}+2 \boldsymbol{\theta} \cdot \mathbf{X}_{T}+\boldsymbol{\theta}^{2} X^{+}, \\
\psi(\zeta) & \Rightarrow\left(\begin{array}{cr}
\mathbf{1} & \mathbf{0} \\
\boldsymbol{\theta} \cdot \boldsymbol{\sigma} \sigma_{z} & \mathbf{1}
\end{array}\right) \psi(\zeta),
\end{aligned}
$$

which leaves $p^{+}, \zeta, \mathcal{F}_{\text {in }}$ and the spinor $\phi$ invariant. We specify the kinematical variables in this frame with a check ( ${ }^{\sim}$ ) accent. Then, $\check{k}^{-}=0, \check{\mathbf{P}}_{f T}=\check{\mathbf{P}}_{i T} \equiv \check{\mathbf{P}}_{T}$ and $\check{\mathbf{p}}_{f T}(\zeta)=\check{\mathbf{p}}_{i T}(\zeta) \equiv \check{\mathbf{p}}_{T}(\zeta)$. Equation (14) rewrites

$$
\left\langle\lambda_{f}|a(\mathbf{k}, \hat{\boldsymbol{\varepsilon}})| \lambda_{i}\right\rangle=\int_{-\infty}^{+\infty} \frac{d \zeta}{2}\left\langle\lambda_{f}|Q(\hat{\boldsymbol{\varepsilon}}, \zeta)| \lambda_{i}\right\rangle e^{i \xi(\zeta)}
$$

with

$$
\begin{gathered}
\xi(\zeta)=\frac{k^{+}}{2} \int_{0}^{\zeta} d \zeta^{\prime} \frac{m^{2}+\check{\mathbf{p}}_{T}^{2}\left(\zeta^{\prime}\right)}{p_{i}^{+} p_{f}^{+}} \\
=C k \cdot\left[X_{i, \mathrm{cl}}(\zeta)-X_{i, \mathrm{cl}}(0)\right], \\
C=p_{i}^{+} / p_{f}^{+},
\end{gathered}
$$

\footnotetext{
${ }^{3}$ This frame is, e.g., the Breit frame of the emitted photon and a photon of the initial wave. Other $\mathbf{k}_{T}=\mathbf{0}$ frames are related to it by a boost along $\hat{\mathbf{z}}$.
}

$$
\begin{aligned}
Q(\hat{\boldsymbol{\varepsilon}}, \zeta)= & \sigma_{z} \hat{\boldsymbol{\varepsilon}}^{*} \cdot \boldsymbol{\sigma} \frac{m+\boldsymbol{\sigma} \cdot \check{\mathbf{p}}_{T}(\zeta) \sigma_{z}}{p_{i}^{+}} \\
& +\frac{m+\sigma_{z} \boldsymbol{\sigma} \cdot \check{\mathbf{p}}_{T}(\zeta)}{p_{f}^{+}} \hat{\boldsymbol{\varepsilon}}^{*} \cdot \boldsymbol{\sigma} \sigma_{z} .
\end{aligned}
$$

Expression (18a) of the phase $\xi(\zeta)$ is derived with the help of Eq. (8). Using (12) and $k^{+} \tau / 2=k \cdot X$, it becomes (18b). Thus, although we use a full quantum treatment, the phase in the integrand of the amplitude $\left\langle\lambda_{f}|a(\mathbf{k}, \hat{\boldsymbol{\varepsilon}})| \lambda_{i}\right\rangle$ is expressed in terms of a classical trajectory associated to the initial Volkov state.

We choose photons of helicity $\Lambda= \pm 1$, of polarization vector $\hat{\boldsymbol{\varepsilon}}=\hat{\boldsymbol{\varepsilon}}(\Lambda)=(\hat{\mathbf{x}}+i \Lambda \hat{\mathbf{y}}) / \sqrt{2}$. Representing helicities only by their signs, Eq. (20) gives

$$
\begin{aligned}
\langle-|Q(+, \zeta)|-\rangle & =\langle+|Q(-, \zeta)|+\rangle^{*} \\
& =\sqrt{2}\left[\check{p}_{x}(\zeta)-i \check{p}_{y}(\zeta)\right] / p_{i}^{+}, \\
\langle+|Q(+, \zeta)|+\rangle & =\langle-|Q(-, \zeta)|-\rangle^{*} \\
= & \sqrt{2}\left[\check{p}_{x}(\zeta)-i \check{p}_{y}(\zeta)\right] / p_{f}^{+}, \\
\langle-|Q(+, \zeta)|+\rangle & =-\langle+|Q(-, \zeta)|-\rangle \\
& =\sqrt{2}\left(m / p_{f}^{+}-m / p_{i}^{+}\right), \\
\langle+|Q(+, \zeta)|-\rangle & =\langle-|Q(-, \zeta)|+\rangle=0 .
\end{aligned}
$$

Gathering Eqs. (17) and (21a)-(21d) we finally obtain

$$
\begin{aligned}
\langle\Lambda / 2|a(\mathbf{k}, \Lambda)| \Lambda / 2\rangle & =C \hat{\boldsymbol{\varepsilon}}^{*}(\Lambda) \cdot \mathbf{J}, \\
\langle-\Lambda / 2|a(\mathbf{k}, \Lambda)|-\Lambda / 2\rangle & =\hat{\boldsymbol{\varepsilon}}^{*}(\Lambda) \cdot \mathbf{J}, \\
\langle-\Lambda / 2|a(\mathbf{k}, \Lambda)| \Lambda / 2\rangle & =\Lambda \frac{C-1}{\sqrt{2}} I, \\
\langle\Lambda / 2|a(\mathbf{k}, \Lambda)|-\Lambda / 2\rangle & =0,
\end{aligned}
$$

where $I$ and $\mathbf{J}$ are expressed in terms of a classical trajectory associated to the initial state:

$$
\left[\begin{array}{l}
I \\
\mathbf{J}
\end{array}\right]=\int_{-\infty}^{+\infty} \frac{d \zeta}{p_{i}^{+}}\left[\begin{array}{c}
m \\
\check{\mathbf{p}}_{T}(\zeta)
\end{array}\right] e^{i \xi(\zeta)},
$$

$\xi(\zeta)$ being given in (18a).

Averaging Eq. (13) over the initial helicity and summing over the final ones gives the unpolarized spectrum,

$$
\frac{d N(\mathbf{k})}{d^{3} \mathbf{k} / \omega}=\frac{\alpha}{8 \pi^{2}}\left\{(C-1)^{2}|I|^{2}+\left(C^{2}+1\right) \mathbf{J} \cdot \mathbf{J}^{*}\right\} .
$$

The factor $C=p_{i}^{+} / p_{f}^{+}>1$ is the recoil correction. The classical radiation formulas are obtained by putting $C=1$, 
$p_{f}^{+}=p_{i}^{+}$in (18a)-(24). As an example of application of Eqs. (22) we give the Stokes parameters of the photon for unpolarized electrons:

$$
\begin{aligned}
& \mathcal{P}_{1}=\mathcal{N}^{-1} C\left(\left|J_{x}\right|^{2}-\left|J_{y}\right|^{2}\right), \\
& \mathcal{P}_{2}=\mathcal{N}^{-1}\left(C^{2}+1\right) \operatorname{Im}\left(J_{x} J_{y}^{*}\right), \\
& \mathcal{P}_{3}=2 \mathcal{N}^{-1} C \operatorname{Re}\left(J_{x} J_{y}^{*}\right), \\
& \mathcal{N}=(1 / 2)\left[(C-1)^{2}|I|^{2}+\left(C^{2}+1\right) \mathbf{J} \cdot \mathbf{J}^{*}\right]
\end{aligned}
$$

$\left(\mathcal{P}_{1}\right.$ and $\mathcal{P}_{3}=$ degree of polarizations along $\hat{\mathbf{x}}$ and $\hat{\mathbf{x}}+\hat{\mathbf{y}}$ respectively, $\mathcal{P}_{2}=$ mean helicity).

\section{A. Notes on the spin properties}

For the hardest photons we have $p_{f}^{+} \ll p_{i}^{+}$, thus the amplitudes (21b) and (21c) are dominating over (21a): the photon helicity takes the sign of the initial electron one, similarly to the bremsstrahlung case.

The helicity-flip amplitude (21c) vanishes for $m=0$ due to chiral invariance.

In the classical theory the photon is fully polarized; one can check that Eq. (25) gives $\mathcal{P}_{1}^{2}+\mathcal{P}_{2}^{2}+\mathcal{P}_{3}^{2}=1$ for $C=1$. For $C>1$ the polarization is only partial: due to the coupling between the electron and photon spins, part of the entropy (lack of information) on the former affects the later.

We can apply Eqs. (13)-(25) to Compton scattering (see Appendix B). One obtains unconventional but simple expressions (B6) and (B7) for the polarized cross section. The denominators $p_{i}^{+}=\left(s-m^{2}\right) /\left(2 \omega_{i}\right)$ and $p_{f}^{+}=\left(m^{2}-u\right) /$ $\left(2 \omega_{i}\right)$ of (21a) and (21b) come from the $s$ and $u$ poles respectively. The absence of the $s$ pole in $(21 \mathrm{~b})$ may be related to the too large total helicity $\Lambda+\lambda_{f}= \pm 3 / 2$ in the s-channel final state. A similar remark holds for the absence of the $u$ pole in (21a). In (21d) both $u$ and $s$ poles are blocked. The null result in this case is nontrivial since it does not ensue from $P$ and $\mathrm{T}$ invariances.

\section{B. Generalization to $\mathbf{a}_{T} \neq \mathbf{0}$ frame}

The $\mathbf{k}_{T}=0$ frame simplifies the calculations but for applications it is more convenient to use a frame not linked to $\mathbf{k}$, for instance a $\mathbf{p}_{T}=0$ frame, where generally $\mathbf{k}_{T} \neq \mathbf{0}$. The $\mathbf{k}_{T}=0$ frame is deduced from it by the Lorentz transformation (16) with $\boldsymbol{\theta}=-\mathbf{k}_{T} / k^{+}$. In this transformation,

$$
\mathbf{p}_{i T} \Rightarrow \check{\mathbf{p}}_{T} \equiv \mathbf{p}_{i, T}-\left(p_{i}^{+} / k^{+}\right) \mathbf{k}_{T},
$$

to be used in formulas (18a), (20), (21a), (21b) and (23).

\section{COMPARISON WITH THE BAÏER-KATKOV FORMULA}

The unpolarized BK formula, as written in, e.g. [5,13], takes the form of Eq. (24) but with

$$
\begin{gathered}
C=\epsilon_{i} / \epsilon_{f} \\
\xi(t) \simeq \frac{\omega}{2} \int_{0}^{t} d t^{\prime} \frac{m^{2}+\mathbf{p}_{\perp}^{2}\left(t^{\prime}\right)}{\epsilon_{i} \epsilon_{f}} . \\
{\left[\begin{array}{l}
I \\
\mathbf{J}
\end{array}\right]=\int_{-\infty}^{+\infty} \frac{d t}{\epsilon_{i}}\left[\begin{array}{c}
m \\
\mathbf{p}_{\perp}(t)
\end{array}\right] e^{i \xi(t)},}
\end{gathered}
$$

instead of (19), (18a) and (23). $\epsilon_{i}=\epsilon_{f}+\omega$ is the initial electron energy. The BK formula is used for the radiation of an ultrarelativistic electron in an arbitrary nonhomogenous field $\mathcal{F}_{\text {in }}=\{\mathbf{E}(t, \mathbf{r}), \mathbf{B}(t, \mathbf{r})\}$, which is not necessarily a plane wave, e.g., a static field. $\mathbf{p}_{\perp}$ instead of $\mathbf{p}_{T}$ is the component perpendicular to $\mathbf{k}$, not to a $z$ axis related to $\mathcal{F}_{\text {in }}$. To summarize, the BK formula is obtained from (18a), (19), (23) and (24) by parametrizing the trajectory with $t$ instead of $\zeta$ and making the replacements $\check{\mathbf{p}}_{T} \Rightarrow \mathbf{p}_{\perp}$ and

$$
k^{+} \Rightarrow 2 \omega, \quad p^{+} \Rightarrow 2 \epsilon, \quad d \zeta \Rightarrow 2 d t .
$$

Similarly, (22a)-(22d) correspond to equations (18a)-(18d) of [10].

If $\mathcal{F}_{\text {in }}$ is a free plane wave, the BK formula becomes exact in the ultrarelativistic limit $\epsilon_{i} \rightarrow \infty$. This is clear in the $\mathbf{k}_{T}=0$ case, where $\mathbf{p}_{\perp} \equiv \check{\mathbf{p}}_{T}, k^{+} \equiv 2 \omega$ and $p^{+} / \epsilon=$ $d \zeta / d t \rightarrow 2$. To generalize this result in the $\mathbf{k}_{T} \neq \mathbf{0}$ case, we cannot invoke a Lorentz transformation because the BK formula is not Lorentz invariant. However, assuming

$$
\epsilon_{i} \omega \gg p_{i} \cdot k
$$

the 4-vectors $p_{i}$ and $k$ are nearly collinear and we still have $k^{+} d \zeta^{\prime} /\left(p_{i}^{+} p_{f}^{+}\right) \simeq \omega d t^{\prime} /\left(\epsilon_{i} \epsilon_{f}\right)$ for Eq. (18a), $p_{i}^{+} / p_{f}^{+} \simeq$ $\epsilon_{i} / \epsilon_{f}$ for Eq. (19) and $d \zeta / p_{i}^{+} \simeq d t / \epsilon_{i}$ for Eq. (23). It remains to compare $\mathbf{p}_{\perp}$ to $\check{\mathbf{p}}_{T}$ defined in (26), measured in their respective transverse planes. The direction of motion of the incoming wave being $-\hat{\mathbf{z}}$, we define $\hat{\mathbf{z}}^{\prime}=\mathbf{k} /|\mathbf{k}|$ and write $\check{\mathbf{p}}_{T}=\check{p}_{x} \hat{\mathbf{x}}+\check{p}_{y} \hat{\mathbf{y}}$ and $\mathbf{p}_{\perp}=p_{x^{\prime}} \hat{\mathbf{x}}^{\prime}+p_{y} \hat{\mathbf{y}}$, where $\hat{\mathbf{y}}=$ $\hat{\mathbf{z}} \times \hat{\mathbf{z}}^{\prime} /\left|\hat{\mathbf{z}} \times \hat{\mathbf{z}}^{\prime}\right|$ is a common $y$ axis, $\hat{\mathbf{x}}=\hat{\mathbf{y}} \times \hat{\mathbf{z}}$ and $\hat{\mathbf{x}}^{\prime}=\hat{\mathbf{y}} \times \hat{\mathbf{z}}^{\prime}$. Then $p_{y}=\check{p}_{y}$ and some calculations gives

$$
p_{x^{\prime}}-\check{p}_{x}=(p \cdot k) k_{x} /\left(\omega k^{+}\right)
$$

In the ultrarelativistic limit, $k^{+} \rightarrow \infty$ while $p \cdot k$ and $\mathbf{k}_{T} / \omega$ stay finite, therefore $p_{x^{\prime}}-\check{p}_{x} \rightarrow 0$. Thus we have $\left(p_{x^{\prime}}, p_{y}\right) \rightarrow\left(\check{p}_{x}, \breve{p}_{y}\right)$, which completes the proof of the exactness of the BK formula, in the ultrarelativistic limit, for an electron crossing a free plane wave.

\section{CASES OF GOOD ACCURACY}

In the case treated in Sec. III the final trajectory $\mathbf{r}_{f}(\zeta)$ is fully determined, up to a space-time translation at constant 
$\zeta$, by the initial one $\mathbf{r}_{i}(\zeta)$ and $\mathbf{k}$. In the $\mathbf{k}_{T}=0$ frame we have indeed $\mathbf{p}_{f T}(\zeta)=\mathbf{p}_{i T}(\zeta)$ and from Eqs. (11) and (12) one has

$$
\begin{aligned}
\mathbf{r}_{f T}(\zeta)-\mathbf{r}_{f T}(0) & =C\left[\mathbf{r}_{i T}(\zeta)-\mathbf{r}_{i T}(0)\right] \\
\tau_{f}(\zeta)-\tau_{f}(0) & =C^{2}\left[\tau_{i}(\zeta)-\tau_{i}(0)\right]
\end{aligned}
$$

This explains why the amplitudes (22) and (23) involves only $\mathbf{r}_{i}(\zeta)$, more precisely $\mathbf{p}_{T}(\zeta)=p^{+} d \mathbf{r}_{i T} / d \zeta$ [there is an equivalent formula involving only $\mathbf{r}_{f}(\zeta)$ ]. Two properties of $\mathcal{F}_{\text {in }}$ play a crucial role: (i) it is a wave packet traveling at the light velocity; both trajectories are forced to traverse it entirely; (ii) this wave is planar, so that $\mathbf{r}_{i}(\zeta)$ and $\mathbf{r}_{f}(\zeta)$ probe the same field.

These properties are not postulated in the original derivation of the BK formula [1]. However the first one is approximately realized for an ultrarelativistic electron in a static field. Indeed, in the initial electron frame, the field becomes almost equivalent to a radiation field (this is the basis of the equivalent photon method). As for the second property, it is also approximately realized if the field does not vary too fast in the transverse directions, more precisely, if the trajectories $\mathbf{r}_{i}(t)$ and $\mathbf{r}_{f}\left(t ; t_{\mathrm{em}}\right)$ explore approximately the same field.

Here are two examples where the BK formula is applicable, at least for $\gamma \gg 1$ : (i) synchrotron radiation; (ii) coherent bremsstrahlung on atomic planes, provided that $\epsilon_{i T}$ and $\epsilon_{f T} \gg U_{0}$, where $\epsilon_{T}=\epsilon \psi^{2} / 2$ is the transverse energy, $\psi$ is the angle between the atomic planes and the electron velocity outside the crystal and $U_{0}$ is the height (for $e^{+}$) or depth (for $e^{-}$) of the Lindhard potential.

\section{A. Application to bremsstrahlung on a single atom}

In the Born approximation, or if the coherence length $l_{c}$ is much bigger than the atom size $r_{a}$, the differential bremsstrahlung cross section takes the factorized form

$$
\frac{\omega d \sigma}{d^{3} \mathbf{k} d^{2} \mathbf{q}_{\perp}}\left(\mathbf{p}_{i}, \mathbf{p}_{f}, \mathbf{k}\right)=\frac{d \sigma_{\mathrm{el}}}{d^{2} \mathbf{q}_{\perp}} \times \frac{d N(\mathbf{k})}{d^{3} \mathbf{k} / \omega}\left(\mathbf{p}_{i}, \mathbf{p}_{f}, \mathbf{k}\right) ;
$$

$\mathbf{q}=\mathbf{p}_{f}+\mathbf{k}-\mathbf{p}_{i}$ is the momentum transfer; $\sigma_{\mathrm{el}}$ is the elastic cross section; $l_{c}$ is given by

$$
\frac{1}{l_{c}} \equiv-q_{\|}=\frac{2 \omega m^{2}}{p_{i}^{+} p_{f}^{+}}-\frac{\mathbf{p}_{i, \perp}^{2}}{p_{i}^{+}}+\frac{\mathbf{p}_{f, \perp}^{2}}{p_{f}^{+}} \sim \frac{\omega}{\gamma_{i} \gamma_{f}} .
$$

The ${ }_{\perp}$ or $\|$ components are relative to $\mathbf{k}$; here $p^{ \pm} \equiv p^{0} \pm p_{\|}$. The last factor of (34) is independent of the atomic potential. It can be calculated with the BK formula applied to the broken trajectory $\mathbf{r}_{i}(t)$ made of two semi-infinite straight lines respectively parallel to $\mathbf{p}_{i}$ and $\mathbf{p}_{\mathrm{el}}^{\prime}$ defined by

$$
\mathbf{p}_{\mathrm{el}}^{\prime}=\left|\mathbf{p}_{i}\right| \frac{\mathbf{p}_{f}+\mathbf{k}}{\left|\mathbf{p}_{f}+\mathbf{k}\right|}
$$

Introducing the vectors $\mathbf{w}=\mathbf{p}_{\mathrm{i}, \perp} / m, \quad \mathbf{w}^{\prime}=\mathbf{p}_{\mathrm{el}, \perp}^{\prime} / m$, Eq. (23) gives

$$
\begin{aligned}
I & =\frac{2 \gamma}{C \omega}\left(\frac{1}{1+\mathbf{w}^{2}}-\frac{1}{1+\mathbf{w}^{\prime 2}}\right), \\
\mathbf{J} & =\frac{2 \gamma}{C \omega}\left(\frac{\mathbf{w}}{1+\mathbf{w}^{2}}-\frac{\mathbf{w}^{\prime}}{1+\mathbf{w}^{\prime 2}}\right) .
\end{aligned}
$$

The cross section obtained from (34), (24) and (37) is equivalent to the QED one in the Born approximation [see Eqs. (93.7) of [4]), in the ultrarelativistic limit. In pure QED, $\mathbf{w}^{\prime}$ is defined as $\mathbf{p}_{f \perp} / m$ but at high energy we have $\mathbf{p}_{\mathrm{el}}^{\prime} \simeq \mathbf{p}_{f}+\mathbf{k}$ so that $\mathbf{p}_{\mathrm{el}, \perp}^{\prime} \simeq \mathbf{p}_{f \perp}$.

Beyond the Born approximation and for $l_{c} \lesssim r_{a}$, the bremsstrahlung amplitude may be calculated in the eikonal approximation (see e.g. [14]). For a given impact parameter b and in a frame where both the atom and the electron are relativistic, the eikonal wave functions are close to the Volkov ones in the equivalent radiation field. The associated classical trajectories $\mathbf{r}_{i}(t)$ and $\mathbf{r}_{f}\left(t ; t_{\mathrm{em}}\right)$ are practically not separated in the region where the field is important. It seems therefore that the BK formula applies to this case. This has been done in [15] for scalar electron and in [16] to study a correction to the factorization theorem.

\section{B. The case of channeling radiation}

The condition of almost uniformity of the field in the transverse directions is not satisfied in channeling radiation. A warning fact is that the spectral lines predicted by the BK formula differ significantly at large $\omega$ from those given by the quantized transverse energy levels [10]. The discrepancy is especially strong in the free-to-bound transitions, because the initial and final trajectories explore quite different fields. Nevertheless the BK formula is used in Monte Carlo simulations of channeling and above-barrier radiations at high energy [5-8]. In [5,6], for example, the trajectory is divided in pieces containing about one or two channeling oscillations and Eq. (29) is integrated on each piece, prolonged upstream and downstream by two semiinfinite lines of uniform motions. Each piece prolonged in this way is equivalent to the trajectory of an electron crossing a thin crystal. Integrations on the semi-infinite lines introduce a spurious infrared divergence, which however keeps the total radiated energy finite and for which a phenomenological cutoff can be fixed by sum rules [17].

The segmentation prevents large separation between $\mathbf{r}_{i}(t)$ and $\mathbf{r}_{f}\left(t ; t_{\mathrm{em}}\right)$ in the equivalent thin crystal, so that these two trajectories explore nearly the same fields, which is the main condition of validity of the BK formula. On the 
other hand it discards the interferences between emission from different pieces, so that the spectral peaks generated by a periodic trajectory are blurred. This is a drawback especially in the case of planar channeling of positrons at medium ( $\lesssim 10 \mathrm{GeV})$ energy, where channeling radiation is quasimonochromatic. However in axial channeling at high energy the spectral peaks become dense enough and the segmentation method just makes a local average of the spectrum. Anyway, at least in the channeling regime where $\omega_{\max } \ll \epsilon$, the total radiated energy is not affected by the segmentation because it is given by the Liénard formula,

$$
W=\frac{2 \alpha}{3 m^{2}} \int_{-\infty}^{\infty} d t\left(F_{\|}^{2}[\mathbf{r}(t)]+\gamma^{2} \mathbf{F}_{\perp}^{2}[\mathbf{r}(t)]\right),
$$

$\mathbf{F}(\mathbf{r})$ being the force acting on the electron, $\|$ and $\perp$ being relative to the electron velocity.

From what we learned in Sec. VA, incoherent bremsstrahlung and its interference with channeling radiation are automatically included if incoherent scatterings are introduced as kinks of the trajectory. This was done in [5-8], where incoherent scatterings were generated at the differential rate $\left(d^{2} \sigma_{\mathrm{el}} / d^{2} \mathbf{q}\right) \times($ Debye-Waller factor $) \times \rho(\mathbf{r})$, where $\rho(\mathbf{r})$ is the local density of scattering centers. The simulation results obtained with the above methods in $[5,6,18,19]$ are in good enough agreement with experiments.

\section{CONCLUSION}

Using the Volkov states we have given an exact derivation of the formula for the photon spectrum emitted by an electron crossing an electromagnetic plane wave, both in the polarized and unpolarized cases. This formula is similar to the Baïer-Katkov formula, but written in terms of lightlike coordinates. In fact the BK formula converges to it in the ultrarelativistic limit.

We explained why the exact formula for an incident plane wave involves only the initial trajectory. The BK formula also has this property, indicating a strong condition for its validity, namely that in the initial rest frame of the electron the field can be approximated by an electromagnetic plane wave; in particular this field should be homogeneous enough in the transverse directions, so that, after emitting a photon, the electron explores approximately the same field as if its motion was not affected by any recoil. This condition is realized in synchrotron radiation, beamstrahlung in future high-luminosity $e^{+} e^{-}$or $e^{-} e^{-}$colliders, Compton back scattering on intense focused laser beam and coherent bremsstrahlung at transverse energies $\gg\left|U_{0}\right|$.

The BK formula also applies to bremsstrahlung on a single atom. It gives a simple derivation, via Eqs. (34), (24) and (37), of the differential cross section in the Born approximation, but can be applied to the non-Born, nonfactorized regime as well. Concerning channeling radiation, the condition of the nearly uniform field in the transverse directions is not fulfilled, but the BK formula can nevertheless be used to get a smooth photon spectrum, after segmenting the trajectories in pieces containing only a few oscillations.

In passing we have noted interesting spin properties and derived unconventional but simple expressions of the Compton amplitudes. Using crossing symmetry, most of our derivations and conclusions can be transposed to the Baïer-Katkov formula for $e^{+} e^{-}$pair creation in external fields. Due to its simplicity and intuitive character, the Baïer-Katkov formula is an invaluable tool in theory and phenomenology of high-energy electromagnetic processes.

\section{APPENDIX A: DERIVATION OF EQS. (13) AND (14)}

The photon spectrum in the radiative transition from state $|i\rangle$ to state $|f\rangle$ can be written in a covariant way:

$$
d N(\hat{\boldsymbol{\varepsilon}})_{f i}=\frac{d^{3} \mathbf{k}}{2 k^{0}(2 \pi)^{3}}\left|\int d^{4} X j_{f i}^{\mu}(X) A_{\mu}^{*}(X)\right|^{2},
$$

where $j_{f i}^{\mu}(X)=-e \bar{\Psi}_{f}(X) \gamma^{\mu} \Psi_{i}(X)$ is the field source, $A_{\mu}(X)=\varepsilon^{\mu} \exp \left(-i k_{\nu} X^{\nu}\right)$ the photon wave function and $\varepsilon^{\mu}$ the polarization 4-vector. Using $d^{4} X=d^{2} \mathbf{X}_{T} d \tau d \zeta / 2$, the gauge $\varepsilon^{\mu}=(0, \hat{\boldsymbol{\varepsilon}})$ and $\Psi(X)$ of the form (3), Eq. (A1) becomes

$$
\begin{aligned}
d N_{f i}(\hat{\boldsymbol{\varepsilon}})= & \frac{\alpha d^{3} \mathbf{k}}{k^{0}(2 \pi)^{2}} \times \mathcal{I} \\
& \times\left|\int \frac{d \zeta}{2} e^{i k^{-} \zeta / 2} \psi_{f} \dagger(\zeta)\left(\hat{\boldsymbol{\varepsilon}}^{*} \cdot \boldsymbol{\alpha}\right) \psi_{i}(\zeta)\right|^{2}
\end{aligned}
$$

with

$$
\begin{aligned}
\mathcal{I}= & \int d \tau d \tau^{\prime} \exp \left\{-i q^{+}\left(\tau^{\prime}-\tau\right) / 2\right\} \\
& \times \int d^{2} \mathbf{X}_{T} d^{2} \mathbf{X}_{T}^{\prime} \exp \left\{i \mathbf{q}_{T} \cdot\left(\mathbf{X}_{T}^{\prime}-\mathbf{X}_{T}\right)\right\}
\end{aligned}
$$

and $q^{+}=p_{f}^{+}+k^{+}-p_{i}^{+}, \mathbf{q}_{T}=\mathbf{P}_{f T}+\mathbf{k}_{T}-\mathbf{P}_{i T}$. Integrating only on $\tau^{\prime}$ and $\mathbf{X}_{T}^{\prime}$ gives

$$
\mathcal{I}=2(2 \pi)^{3} \delta\left(q^{+}\right) \delta^{2}\left(\mathbf{q}_{T}\right) \int d \tau d^{2} \mathbf{X}_{T}
$$

The delta functions express the conservation laws (15). The remaining integral in (A4) is the total area $\times$ duration of the incident wave front. It is infinite, as the timeintegrated total flux $\left(J_{i}^{+} / 2\right) \int d \tau d^{2} \mathbf{X}_{T}$ of initial electron across the wave front, with $J_{i}^{+}=2$ according to (10). Thus, removing the infinite factor $\int d \tau d^{2} \mathbf{X}_{T}$ and keeping

$$
\mathcal{I}=2(2 \pi)^{3} \delta\left(p_{f}^{+}+k^{+}-p_{i}^{+}\right) \delta^{2}\left(\mathbf{P}_{f T}+\mathbf{k}_{T}-\mathbf{P}_{i T}\right),
$$


the expression (A2) becomes the differential photon yield per incident electron.

We now sum over the final electron state $|f\rangle$, using the completeness relation

$\sum_{f}|f\rangle\left\langle f\left|=\sum_{\lambda_{f}} \int \frac{d p_{f}^{+} d^{2} \mathbf{P}_{f T}}{J_{f}^{+}(2 \pi)^{3}}\right| p_{f}^{+}, \mathbf{P}_{f T}, \lambda_{f}\right\rangle\left\langle p_{f}^{+}, \mathbf{P}_{f T}, \lambda_{f}\right|$,

associated to the orthogonality relation (9), with $J_{f}^{+}=2$. The integrations over $p_{f}^{+}$and $\mathbf{P}_{f T}$ are trivial owing to (A5). Equations (13) and (14) are thus obtained.

\section{APPENDIX B: APPLICATION TO COMPTON SCATTERING}

We consider the reaction $e_{i}^{-}\left(P_{i}\right)+\gamma_{i}\left(k_{i}\right) \rightarrow e_{f}^{-}\left(P_{f}\right)+$ $\gamma(k)$, where $k \equiv k_{f}$. The incoming photon can be described by the classical field

$$
\mathbf{A}_{T}(\zeta)=\mathcal{A} \operatorname{Re}\left[\hat{\boldsymbol{\varepsilon}}_{i} \exp \left(-i \omega_{i} \zeta\right)\right]
$$

with $\hat{\boldsymbol{\varepsilon}}_{i} \cdot \hat{\boldsymbol{\varepsilon}}_{i}^{*}=1$. We first place ourselves a frame where $\mathbf{P}_{i T}=\mathbf{0}, \mathbf{P}_{f, T}=-\mathbf{k}_{T}, \mathbf{p}_{i T}(\zeta)=e \mathbf{A}_{\mathrm{T}}(\zeta)$, e.g., the c.m. frame or the $e_{i}^{-}$rest frame. Applying (26),

$$
\begin{gathered}
\check{\mathbf{P}}_{T}=-\left(p_{i}^{+} / k^{+}\right) \mathbf{k}_{T}, \\
\check{\mathbf{p}}_{T}(\zeta)=\check{\mathbf{P}}_{T}+e \mathbf{A}_{T}(\zeta) .
\end{gathered}
$$

Calculating (18) and (23) to first order in $e \mathcal{A}$, we obtain

$$
\left[\begin{array}{l}
I \\
\mathbf{J}
\end{array}\right]=2 \pi \delta\left(\frac{m^{2}+\check{\mathbf{P}}_{T}^{2}}{2 p_{i}^{+} p_{f}^{+}} k^{+}-\omega_{i}\right)\left[\begin{array}{l}
I^{\prime} \\
\mathbf{J}^{\prime}
\end{array}\right]
$$

with

$$
\left[\begin{array}{l}
I^{\prime} \\
\mathbf{J}^{\prime}
\end{array}\right]=\frac{-e \mathcal{A} k^{+}}{p_{i}^{+}}\left[\begin{array}{c}
c m \check{\mathbf{P}}_{T} \cdot \hat{\boldsymbol{\varepsilon}}_{i} \\
c\left(\check{\mathbf{P}}_{T} \cdot \hat{\boldsymbol{\varepsilon}}_{i}\right) \check{\mathbf{P}}_{T}-\hat{\boldsymbol{\varepsilon}}_{i} / 2
\end{array}\right]
$$

and $c=k^{+} /\left[2 \omega_{i} p_{i}^{+} p_{f}^{+}\right]$. Integrating (13) with (22) and (23) over $k^{+}$gives the differential photon spectrum $d N(\mathbf{k}) / d^{2} \mathbf{k}_{T}$ of the scattered photon. This is done in analogy with (A3) and (A4): the square of the $2 \pi \delta(\cdots)$ factor of (B3) is replaced by $2 \pi \delta(\cdots) \times \int d \zeta$. Integrating the delta function over $k^{+}$(noting $p_{f}^{+}=p_{i}^{+}-k^{+}$) yields a factor $2\left(p_{f}^{+}\right)^{2} /\left(m^{2}+\check{\mathbf{P}}_{T}^{2}\right)$. Removing $\int d \zeta$ and dividing by the incident photon density

$$
d N_{\text {phot }} /\left(d^{2} \mathbf{X}_{\mathrm{T}} d \zeta\right)=\mathcal{A}^{2} \omega_{i} / 2
$$

we obtain the fully polarized Compton cross section in the form

$$
\frac{d \sigma^{\mathrm{pol}}}{d^{2} \mathbf{w}}=\frac{4 \alpha^{2}}{m^{2}} \times \frac{\left|\left\langle\hat{\boldsymbol{\varepsilon}}, \lambda_{f}|M(\tau)| \hat{\boldsymbol{\varepsilon}}_{i}, \lambda_{i}\right\rangle\right|^{2}}{C^{3}\left(1+\mathbf{w}^{2}\right)^{4}}
$$

where $\mathbf{w}=\check{\mathbf{P}}_{T} / m$ and $C=\left(p_{i} \cdot k_{i}\right) /\left(p_{i} \cdot k\right)$ is linked to $\mathbf{w}$ by $(C-1)\left(1+\mathbf{w}^{2}\right)=2 p_{i} \cdot k_{i} / m^{2}$. $\mathbf{w}^{2}$ ranges from 0 for backward scattering to $\infty$ for forward scattering. Introducing the complex variable $\underline{w}=w_{x}+i w_{y}$, the matrix elements of $M$ for circular polarizations $\mathrm{R}$ or $\mathrm{L}$ of the photons and helicities $\pm 1 / 2$ of the electrons have the simple forms

$$
\begin{aligned}
\langle L,-|M| L,-\rangle & =\langle R,+|M| R,+\rangle^{*}=C \underline{w}^{2}, \\
\langle L,+|M| L,+\rangle & =\langle R,-|M| R,-\rangle^{*}=\underline{w}^{2}, \\
\langle R,-|M| L,+\rangle & =\langle R,-|M| R,+\rangle^{*}=(C-1) \underline{w}, \\
\langle L,+|M| L,-\rangle & =\langle L,+|M| R,-\rangle^{*}=(1-C) \underline{w}, \\
\langle R,+|M| L,+\rangle & =\langle L,-|M| R,-\rangle=-C, \\
\langle R,-|M| L,-\rangle & =\langle L,+|M| R,+\rangle=-1, \\
\langle R,+|M| L,-\rangle & =\langle L,-|M| R,+\rangle=0 .
\end{aligned}
$$

The unpolarized Compton cross section

$$
\frac{2 \alpha^{2}}{m^{2}} \int d^{2} \mathbf{w} \frac{\left(1+C^{2}\right)\left(1+|\mathbf{w}|^{4}\right)+2(C-1)^{2} \mathbf{w}^{2}}{C^{3}\left(1+\mathbf{w}^{2}\right)^{4}}
$$

agrees the standard result found, e.g., in [4].

[1] V. N. Baier and V. M. Katkov, Processes involved in the motion of high energy particles in a magnetic field, Zh. Eksp. Teor. Fiz. 53, 1478 (1967) [Sov. Phys. JETP 26, 854 (1968)]; Quasiclassical theory of bremsstrahlung by relativistic particles, Zh. Eksp. Teor. Fiz.55, 1542 (1968) [Sov. Phys. JETP28, 807 (1969)].

[2] V. N. Baier, V. M. Katkov, and V. M. Strakhovenko, Electromagnetic Processes at High Energies in Oriented Crystals (World Scientific, Singapore, 1998).

[3] J. D. Jackson, Classical Electrodynamics (Wiley, NY, 1999).

[4] V. B. Berestetskii, E. M. Lifshitz, and L. P. Pitaevskii, Quantum Electrodynamics (Pergamon Press, Oxford, 1982).

[5] X. Artru, A simulation code for channeling radiation by ultrarelativistic electrons or positrons, Nucl. Instrum. Methods Phys. Res., Sect. B 48, 278 (1990).

[6] X. Artru, Channeling radiation: Theory, semi-classical simulations, Radiat. Eff. Defects Solids 130-132, 415 (1994).

[7] L. Bandiera, V. Haurylavets, and V. V. Tikhomirov, Compact electromagnetic calorimeters based on oriented 
scintillator crystals, Nucl. Instrum. Methods Phys. Res., Sect. A, (2018).

[8] V. Guidi, L. Bandiera, and V. V. Tikhomirov, Radiation generated by single and multiple volume reflection of ultrarelativistic electrons and positrons in bent crystals, Phys. Rev. A 86, 042903 (2012).

[9] X. Artru, G. Bignon, and T. Qasmi, Side-slipping of a radiating particle, Problems At. Sci. Technol. 6, 98 (2001).

[10] X. Artru, Classical and quantum phenomenology in radiation by relativistic electrons in matter or in external fields, Nucl. Instrum. Methods Phys. Res., Sect. B 355, 11 (2015).

[11] D. M. Volkov, Über eine Klasse von Lösungen der Diracschen Gleichung, Z. Phys. 94, 250 (1935).

[12] V. I. Ritus, Quantum effects of the interaction of elementary particles with an intense electromagnetic field, J. Sov. Laser Res. 6, 497 (1985).

[13] A. I. Akhiezer and N. F. Shul'ga, High-Energy Electrodynamics in Matter (Gordon and Breach, New York, 1996).

[14] R. N. Lee, A. I. Milstein, V. M. Strakhovenko, and O. Ya. Schwarz, Coulomb corrections to bremsstrahlung in the electric field of a heavy atom at high energies, J. Exp. Theor. Phys. 100, 1 (2005).

[15] A. I. Akhiezer and N. F. Shul'ga, Canonical transformations in the quantum theory of radiation at high energies and the recoil effect under radiation, Phys. Lett. A 158, 275 (1991).

[16] M. V. Bondarenco, Next-to-leading order correction to the factorization limit of the radiation spectrum, Phys. Rev. D 96, 076009 (2017).

[17] X. Artru, Filtered sum rules for synchrotron radiation in nonuniform field. Application to channeling radiation, Radiat. Eff. Defects Solids 116, 293 (1991).

[18] L. Bandiera, E. Bagli, V. Guidi, A. Mazzolari, A. Berra, D. Lietti, M. Prest, E. Vallazza, D. De Salvador, and V. Tikhomirov, Broad and Intense Radiation Accompanying Multiple Volume Reflection of Ultrarelativistic Electrons in a Bent Crystal, Phys. Rev. Lett. 111, 255502 (2013).

[19] L. Bandiera, V. V. Tikhomirov, M. Romagnoni, N. Argiolas, E. Bagli, G. Ballerini, A. Berra, C. Brizzolari, R. Camattari et al., Strong Reduction of the Effective Radiation Length in an Axially Oriented Scintillator Crystal, Phys. Rev. Lett. 121, 021603 (2018). 